\title{
NIR Spectroscopic Determination of Polyphenol Content in Teas and Tea Extract at $2142 \mathrm{~nm}$
}

\author{
Mahasen Ranatunga, ${ }^{1}$ Yasuhiro Uwadaira, ${ }^{2 \#}$ Akifumi Ikehata, ${ }^{2}$ and Hidekazu Ito ${ }^{2 *}$ \\ ${ }^{1}$ Tea Research Institute of Sri Lanka (TRISL), Talawakelle 22100, Sri Lanka \\ ${ }^{2}$ Food Research Institute, National Agriculture and Food Research Organization (NARO), \\ 2-1-12, Kannondai, Tsukuba, Ibaraki 305-8642, Japan
}

(Received September 10, 2020; accepted February 12, 2021; online published February 25, 2021)

Keywords: NIR, transmittance-reflectance cell, total polyphenol (TPP), PLS regression analysis, correlation spectrum

Various teas, a tea extract, and a catechin mixture reagent were measured in transmittancereflectance mode using visible and NIR (Vis-NIR) spectroscopy. Total polyphenol (TPP) content in the tea samples was determined using Vis-NIR spectra. Then, reference TPP content was determined by the colorimetric ferrous tartrate method. Partial least squares (PLS) regression, a method of multivariate analysis, was performed in combination with various wavelength ranges and spectral pretreatments. Usually, multivariate analyses in which many independent variables are applied have been used for NIR data to obtain high accuracy for the determination. Nevertheless, surprisingly, only $2142 \mathrm{~nm}$, which has high sensitivity and is in the NIR region, was identified for the determination of TPP content in various teas and tea extracts based on regression coefficient values, selectivity ratio (SR) values of the best PLS model [correlation coefficient $(R=0.98, n=69)$ ], correlation coefficients (a correlation spectrum) between the reference TPP content and Savitzky-Golay smoothing with the second derivatives (SG2D) with standard normal variate (SNV) preprocessing of raw spectra, and the peaks of the raw spectra and their SG2D with SNV. The R value between SG2D with SNV at $2142 \mathrm{~nm}$ and reference TPP content was $0.96(n=69)$.

\section{Introduction}

Spectroscopy, the measurement of light intensity absorbed by a sample as a function of wavelength, is an analytical tool for identifying a wide array of compounds in agricultural products. In particular, near infrared (NIR, 800-2500 nm) spectroscopy is a new technique that exploits the region neigboring 2500 to $25000 \mathrm{~nm}\left(4000\right.$ to $\left.400 \mathrm{~cm}^{-1}\right)$ used in IR spectroscopy, and can provide rapid, accurate, and non-destructive analyses of agricultural products on site or online without wet chemical analysis. NIR spectroscopy, also named overtone vibrational spectroscopy, is especially suitable for rapidly measuring total amounts of water, protein, lipid, and so on, in food. ${ }^{(1)}$ However, it is very difficult to determine their total amounts using only one NIR wavelength as an independent variable, because single regression analyses, in which

\footnotetext{
\#Present address: ABeam consulting Ltd, Marunouchi Eiraku Building, 1-4-1 Marunouchi, Chiyoda-ku, Tokyo, Japan (Both M. Ranatunga and Y. Uwadaira contributed equally to this work.)

*Corresponding author: e-mail: hito7@affrc.go.jp

https://doi.org/10.18494/SAM.2021.3087

ISSN 0914-4935 (C) MYU K.K. https://myukk.org/
} 
only one independent variable is applied, have low accuracy. Therefore, multivariate analyses, in which many independent variables are applied, have been used for NIR data to obtain high accuracy for their determination.

Concrete examples are given below. The health benefits of catechins or polyphenol in teas have been reported in numerous publications. ${ }^{(2-5)}$ Catechins are the main components of the total polyphenol (TPP) in teas. ${ }^{(6)}$ Schulz et al. reported the determination of TPP in green teas using NIR spectroscopy. ${ }^{(7)}$ Other studies have also shown the use of NIR spectroscopy for determining TPP or catechins in green (color) teas. ${ }^{(7-14)}$ Ren et al. and Panigrahi et al. reported the use of NIR spectroscopy for determining TPP in black teas. ${ }^{(15,16)}$ Schulz et al., Chen et al., Sinija and Mishra, and Wang et al. used wavelength ranges of 1108 to 2490, 909 to 2632, 800 to 2500, and 1000 to $2500 \mathrm{~nm}$, respectively, for TPP determination in green teas. ${ }^{(7-9,12)}$ Bian et al. identified 1131, 1654, 1666, 1738, and $1752 \mathrm{~nm}$ as bands related to the absorption of TPP for leaf powders as well as fresh leaves. ${ }^{(10)}$ Moreover, Bian et al. reported that $1648 \mathrm{~nm}$ can be linked to the absorption feature of phenolic acid for tea powder and leaves. ${ }^{(11)}$ Schulz et al., Luypaert et al., and Lee et al. used wavelength ranges of 1108 to 2490, 1100 to 2500, and 1100 to $2500 \mathrm{~nm}$, respectively, for catechin determination in green teas. ${ }^{(7,13,14)}$ Ren et al. and Panigrahi et al. used wavelength ranges of 800 to 2500 and 400 to $2447 \mathrm{~nm}$, respectively, for TPP determination in black teas. ${ }^{(15,16)}$

Thus, many wavelengths can already be used for TPP or catechin determination in green or black teas. Because we cannot know a few wavelengths as independent variables for TPP determination in teas, these NIR methods have low potential for practical use; we would have to specify a few independent variables with pinpoint accuracy. Although Panigrahi et al. achieved high accuracy $\left(R^{2}=0.96\right)$ by using 400 to $2447 \mathrm{~nm}$ for calibrating TPP in black teas, their accuracy was low $\left(R^{2}=0.79\right)$ for TPP validation. ${ }^{(16)}$ Moreover, Schulz et al. reported a lower correlation $\left(R^{2}=0.67\right)$ for NIR spectroscopic measurements (1108 to $\left.2490 \mathrm{~nm}\right)$ of TPP in dried green tea leaves. ${ }^{(7)}$ On the other hand, the soluble solids of tomatoes and strawberries, which have high water content, can be non-destructively determined using only three wavelengths in the short NIR range. ${ }^{(17)}$ If samples are dry, the accuracy could be higher because no large peaks associated with water absorption will appear in their NIR spectra.

As mentioned above, black and green teas have been examined separately, and different models have been developed with many independent variables. Also, the visible (Vis) region has been evaluated for the rapid analysis of green and black teas, ${ }^{(10,16)}$ which may be effective for determining TPP in teas because the Vis region is sensitive to TPP, as shown by Pan et al. with a black tea infusion. ${ }^{(18)}$ Moreover, tea extract with a high TPP content may help to provide better results for the determination of TPP in a new transmittance-reflectance mode using visible and NIR (Vis-NIR) spectroscopy.

Regarding multivariate analysis, the partial least squares (PLS) algorithm has been used with many independent variables without specifying a few wavelengths for the determination. ${ }^{(7-16)}$ PLS regression analyses can be performed in combination with different wavelength ranges and spectral pretreatments to specify an NIR range for TPP determination. ${ }^{(19)}$ Also, the combination of new PLS analyses and the correlation coefficients between reference TPP values and VisNIR spectra (a correlation spectrum) may specify a wavelength for TPP determination. 
Therefore, we measured various teas and tea extracts in a new transmittance-reflectance mode using a Vis-NIR spectrophotometer and analyzed the data using the new PLS analyses and correlation spectrum (Fig. 1). As a result, we were unexpectedly able to specify a new key wavelength $(2142 \mathrm{~nm})$ for TPP determination.

\section{Materials and Methods}

\subsection{Samples}

A total of 69 samples were used, including black teas from the Tea Research Institute of Sri Lanka (TRISL), commercial black, green, oolong, Kamairi, Pu'er, Houji, and Sunrouge teas, tea extracts, and a tea extract containing dextrin. The samples are given in Table 1.

\subsection{Sample preparation}

About $10 \mathrm{~g}$ of each sample was ground using a tea grinder (National MX-X57; Panasonic Corporation, Osaka, Japan) for $30 \mathrm{~s}$ and passed through a 35-mesh sieve (pore size of $500 \mu \mathrm{m}$ ) using a mechanical sieve shaker (MVS-1; As One, Osaka, Japan). The tea extracts and tea extract containing dextrin, which have fine particles, were used after sieving without grinding to measure the Vis-NIR spectrum and determine the reference TPP content.

\subsection{Measurement of Vis-NIR spectra}

The temperature in the laboratory was maintained near $24{ }^{\circ} \mathrm{C}$. Vis-NIR spectra (400-2498 nm) were acquired for $0.3 \mathrm{~g}$ of each sample and a catechin mixture reagent (green tea; Wako Pure Chemical Industries Ltd., Osaka, Japan) in transmittance-reflectance mode with a sample cell, in which a gold reflection plate was set with a $0.4 \mathrm{~mm}$ total path length, using a Foss XDS Rapid Content Analyzer (Foss Analytical Solutions, Hillerod, Denmark). For each sample, 32 scans were averaged at $2 \mathrm{~nm}$ intervals. WinISI and ISIscan software were used (Foss Analytical AB, Hoganas, Sweden).

Table 1

Set of tea samples $(n=69)$.

\begin{tabular}{lc}
\hline Sample & Total samples \\
\hline Black tea & 39 \\
Green tea & 19 \\
Tea extract & 2 \\
Tea extract containing dextrin & 1 \\
Oolong tea & 3 \\
Pu'er tea & 1 \\
Kamairi tea & 2 \\
Houji tea & 1 \\
Sunrouge tea & 1 \\
\hline
\end{tabular}




\subsection{Reference TPP content}

Reference values of TPP content were determined using the colorimetric ferrous tartrate method described by Iwasa and Torii. ${ }^{(20)}$ About $50 \mathrm{mg}$ of each sample was extracted in $40 \mathrm{~mL}$ of distilled water for 30 min using a water bath set at $80 \pm 2{ }^{\circ} \mathrm{C}$ (Elma $30 \mathrm{H}$; Elma Hans Schmidbauer GmbH \& Co, Singen, Germany). Then, the extract was mixed well for $5 \mathrm{~min}$. The extract was cooled at room temperature and filtered through a disposable filter with a $0.45 \mu \mathrm{m}$ pore size (Advantec Dismic $25 \mathrm{hp}$; Toyo Roshi Kaisha Ltd., Tokyo, Japan). Gallic acid (gallic acid monohydrate; Wako) was used to prepare a series of standard solutions $(10,20,30$, 40 , and $50 \mu \mathrm{g} \mathrm{mL}^{-1}$ ). A dye solution was prepared by dissolving $100 \mathrm{mg}$ of ferrous sulphate heptahydrate (Wako) and $500 \mathrm{mg}$ of potassium sodium tartrate tetrahydrate (special grade; Kanto Chemicals Co. Inc., Tokyo, Japan) in $100 \mathrm{~mL}$ of distilled water. Phosphate buffer was prepared with a concentration of $66 \mathrm{mM}$ at $\mathrm{pH} 7.5$ by mixing disodium hydrogen phosphate (special grade; Kanto Chemicals) and potassium dihydrogen phosphate (special grade; Kokusan Kagaku Chemicals Co., Ltd.). Filtered tea extract $(5 \mathrm{~mL})$ was mixed with dye solution $(5 \mathrm{~mL})$ and phosphate buffer $(15 \mathrm{~mL})$. Sample absorbance was measured at $540 \mathrm{~nm}$ using a UV-NIR spectrophotometer (Solid Lambda CCD; Spectra Co-op, Tokyo, Japan), and the absorbance of each sample blank solution with the dye solution replaced with distilled water was subtracted. TPP content is expressed as $\mathrm{w} / \mathrm{w} \%$.

\subsection{Data analysis of reference TPP content}

For the 69 samples of teas and tea extract, descriptive statistical parameters (mean, standard error, standard deviation, and coefficient of variation) of their reference TPP content were calculated.

\subsection{Preprocessing spectra and multivariate analysis}

Spectral data were acquired in the range of $400-2498 \mathrm{~nm}$ at $2 \mathrm{~nm}$ intervals. To reduce the systematic noise in the spectra and enhance the contribution of the chemical components, different spectral preprocessing methods were applied, including the standard normal variate (SNV), ${ }^{(21)}$ autoscaling (AS), first and second derivatives (1D, 2D), and Savitzky-Golay smoothing (SG) with a window size of 11 points and a polynomial of order 2. ${ }^{(22)}$ Mean centering was always applied to each model. The following wavelength ranges were used for the analyses: 400-800, 400-1100, 400-1700, 400-2200, 400-2498, 800-1100, 800-2498, 9001700, 1100-1700, 1100-2498, 1700-2200, 1700-2498, 1850-2350, 2000-2450, 2000-2498, and 2100-2450 nm. Spectral models were developed to predict TPP content using PLS regression analyses, and the number of latent variables required for each PLS regression model was calculated by the leave-one-out cross-validation approach. Thus, PLS regression analyses were performed in combination with each wavelength range and different spectral pretreatments using the 'plsropt' package in R. ${ }^{(19)}$ The best PLS model was selected using the correlation coefficient $(R)$, with the ratio of performance deviation (RPD) used for validation, where RPD is the standard deviation of the reference TPP content divided by the standard error of crossvalidation (SECV). 


\subsection{Identification of optimal wavelength for predicting TPP}

An optimal wavelength for determining TPP was identified using the regression coefficients, the selectivity ratio (SR) values of the best PLS model, the R values between the reference TPP content and SG2D with SNV preprocessing of raw spectra (the correlation spectrum), and the peaks of the raw spectra and SG2D with SNV. SR is the ratio of the explained variance of each variable to the residual variance.

\section{Results}

\subsection{Reference TPP content of samples}

Table 2 gives the descriptive statistics of the reference TPP content in the sample set $(n=$ 69). Reference TPP content ranged from 3.5 to $32.1 \%$ with a mean value of $10.2 \%$, standard deviation of $5.0 \%$, and coefficient of variation of $49.0 \%$.

\subsection{Raw spectra}

Figure 2 shows averaged raw spectra of black, green, oolong, Pu'er, Kamairi, Houji, and Sunrouge teas, tea extract, tea extract containing dextrin, and catechin reagent. Black tea showed higher absorption than green tea. Oolong tea showed absorption between those of green and black teas but higher absorption at long wavelengths. Clear absorption peaks are observed at 476, 672, 1460, 1930, and $2140 \mathrm{~nm}$. The peak at $2140 \mathrm{~nm}$ was broad, spanning 2080-2210 nm.

\subsection{SG2D spectra with SNV preprocessing of raw spectra}

A lower 2D value indicates stronger absorption. Clear absorption peaks are observed at 674, 1438, 1914, and $2142 \mathrm{~nm}$ (Figs. 3 and 4), corresponding to absorption at 672, 1460, 1930, and $2140 \mathrm{~nm}$ in the raw spectra, respectively (Fig. 2). The catechin reagent has the lowest peak values at 674, 1132, 1438, 1660, 1914, 2142, and $2260 \mathrm{~nm}$ (Fig. 4).

\subsection{PLS modeling in combination with wavelength ranges and preprocessing spectra}

The models used to determine TPP content via Vis-NIR spectroscopy were based on PLS regression analyses. Different combinations of wavelength ranges and spectral preprocessing

Table 2

Descriptive statistical parameters for reference TPP content of the sample set.

\begin{tabular}{lc}
\hline Parameter & Values \\
\hline No. of samples & 69 \\
Minimum value (\%) & 3.5 \\
Maximum value (\%) & 32.1 \\
Range (\%) & 28.6 \\
Mean \pm standard error & $10.2 \pm 0.6$ \\
Standard deviation (\%) & 5.0 \\
Coefficient of variation (\%) & 49.0 \\
\hline
\end{tabular}




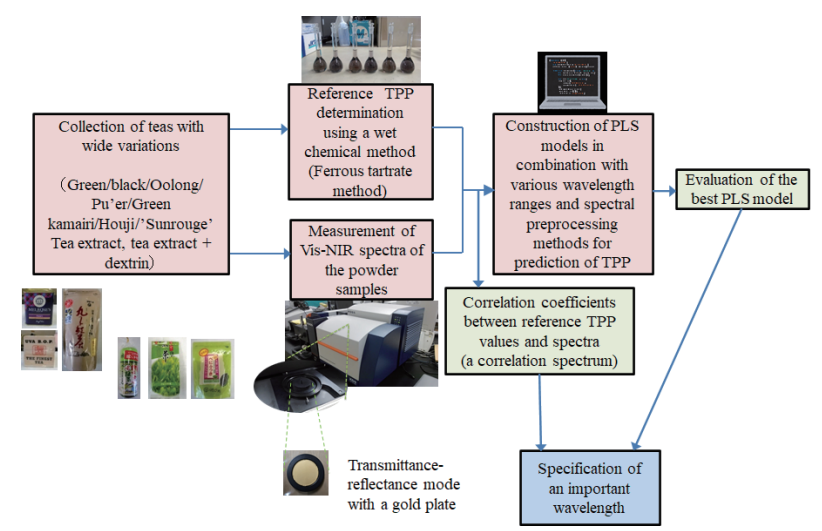

Fig. 1. (Color) Experimental setup used in this study.

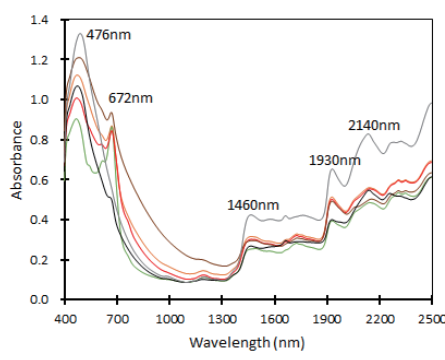

(a)

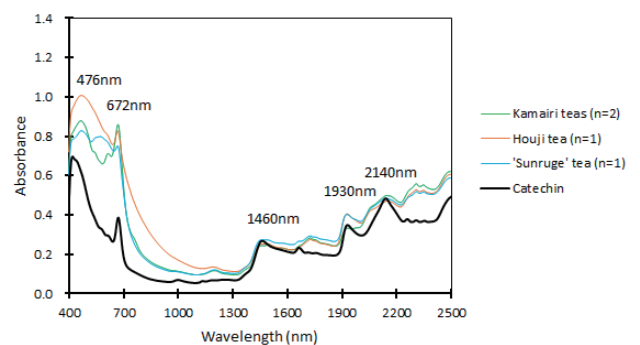

(b)

Fig. 2. (Color) Average raw spectra (400 to $2498 \mathrm{~nm}$ ): (a) black, green, oolong, and Pu'er teas, tea extract, and tea extract containing dextrin. (b) Kamairi, Houji, and Sunrouge teas and catechin reagent.

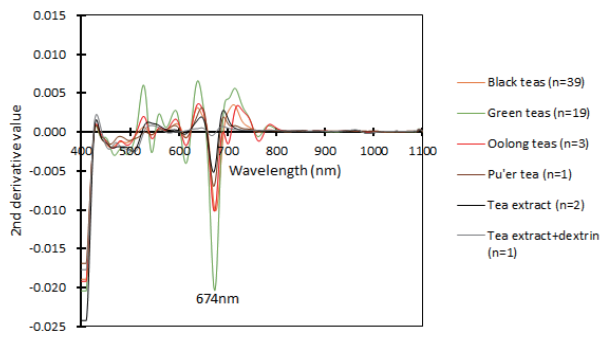

(a)

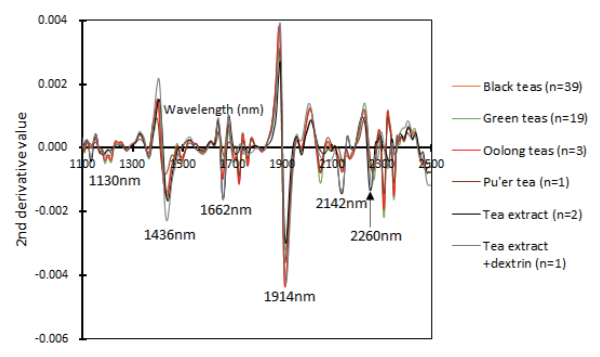

(b)

Fig. 3. (Color) Average second derivative spectra with SNV: (a) 400 to $1100 \mathrm{~nm}$, (b) 1100 to $2498 \mathrm{~nm}$. (Samples: black, green, oolong, and Pu'er teas, tea extract, and tea extract containing dextrin)

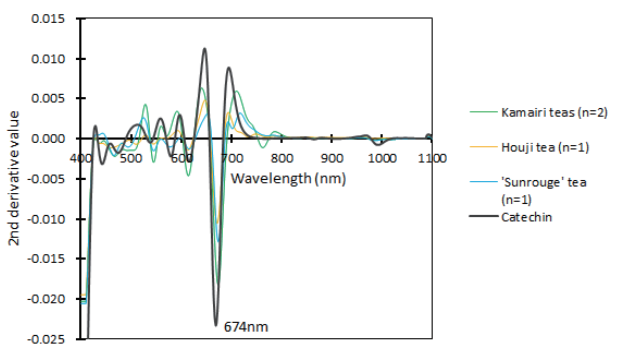

(a)

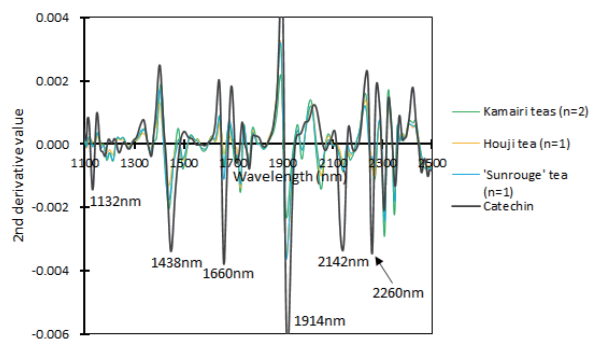

(b)

Fig. 4. (Color) Average second derivative spectra with SNV: (a) 400 to $1100 \mathrm{~nm}$, (b) 1100 to $2498 \mathrm{~nm}$. (Samples: Kamairi, Houji, and Sunrouge teas and catechin reagent) 
methods were compared. The best PLS model was selected for the wavelength range of 2000-2498 nm after spectral preprocessing via SNV and SG2D with SNV. The model has seven latent variables and gave the highest $R$ value of 0.98 , the highest RPD of 4.47, the lowest SECV of 1.12, a slope of 0.922 (near 1), and a low bias of 0.043 (near zero) for the validation (Table 3). Figure 5 shows the relationship between the reference and NIRcalculated TPP values for the best PLS model.

The regression coefficients of the best PLS model are shown in Fig. 6(a). The lowest regression coefficient value (-444.6) was observed at $2144 \mathrm{~nm}$, corresponding to SG2D with SNV. Figure 6(b) shows the SR values of the best PLS model. The highest SR value of 11.1 was observed at $2144 \mathrm{~nm}$.

Table 3

Best PLS model for predicting TPP in various teas and tea extract.

\begin{tabular}{|c|c|c|c|c|c|c|c|}
\hline Wavelength (nm) & Preprocessing method & $\mathrm{N}$ & & & alidatio & & \\
\hline \multirow{2}{*}{ 2000-2498 } & \multirow{2}{*}{$\mathrm{SNV}+\mathrm{SG} 2 \mathrm{D}$} & \multirow{2}{*}{7} & $\mathrm{R}$ & RPD & SECV & Slope & Bias \\
\hline & & & 0.98 & 4.47 & 1.12 & 0.922 & 0.043 \\
\hline
\end{tabular}

SNV: Standard normal variate, SG2D: Savitzky-Golay smoothing with 2nd derivative, N: number of PLS components, R: correlation coefficient for validation, RPD: ratio of performance deviation for validation, SECV: standard error of crossvalidation.

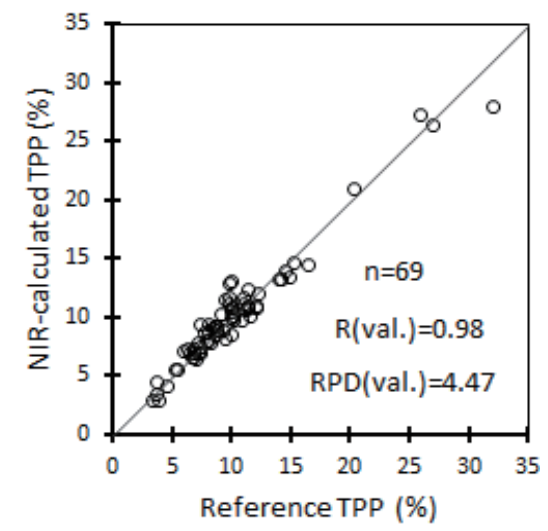

Fig. 5. Plots of reference vs NIR (2000 to $2498 \mathrm{~nm}$, the best PLS model) calculated for TPP.

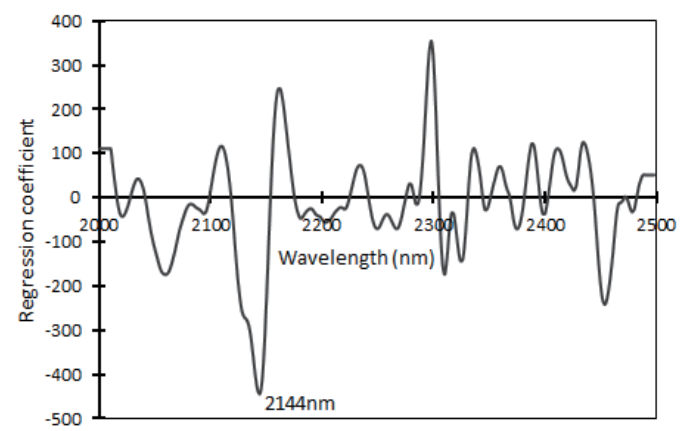

(a)

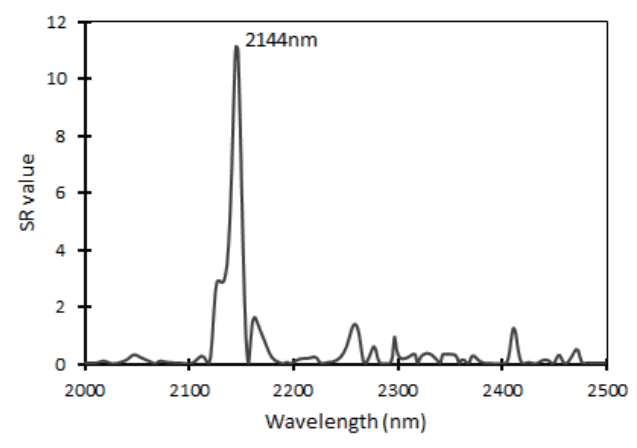

(b)

Fig. 6. Best PLS model. (a) Regression coefficients, (b) SR values. 


\subsection{Correlation coefficients between reference TPP content and SG2D values with SNV}

Figure 7 shows a correlation spectrum for the reference TPP content and SG2D with SNV preprocessing of raw spectra. The lowest negative $R$ value $(-0.96, n=69)$ was observed at $2142 \mathrm{~nm}$. Figure 8 shows plots of reference TPP vs TPP calibrated at $2142 \mathrm{~nm}$ for SG2D with SNV.

\section{Discussion}

\subsection{Reference TPP content of samples}

The Folin-Ciocalteu and ferrous tartrate methods have been used to determine reference TPP content in tea. ${ }^{(7-12,15,16,18)}$ However, a reducing substance, L-ascorbic acid, reacts with the dye solution when TPP content is quantified by the Folin-Ciocalteu method. ${ }^{(23,24)}$ Bian et al. used ferrous tartrate colorimetry to determine the reference TPP concentration, and the VisNIR results showed a correlation of $R^{2}=0.97$ between the reference and calculated TPP concentrations ( $n=56,146.62$ to $294.98 \mathrm{mg} \mathrm{g}^{-1}$ ) in young green tea powder. ${ }^{(10)}$ Schulz et al. reported a lower correlation $\left(R^{2}=0.67\right)$ for NIR spectroscopic measurements of TPP (60.8 to $199.8 \mathrm{mg} \mathrm{g}^{-1}$ ) in dried green tea leaves, which might be due to the lack of specificity in the colorimetric reference method. They used the Folin-Ciocalteu method. ${ }^{(7)}$ Therefore, we used the ferrous tartrate method.

Studies conducted using black teas have reported lower TPP ranges than those using green teas. Ren et al. and Panigrahi et al. reported TPP ranges of 4.2-20.5 and 7.6-17.2\%, respectively, for black tea samples. ${ }^{(15,16)}$ By contrast, higher TPP ranges of 19.2-30.2 and 22.0-30.7\% were reported for green tea samples by Chen et al. and Wang et al., respectively. ${ }^{(8,12)}$ Thus, we were able to obtain a wide range of reference TPP contents for tea samples. In addition, the tea extract samples covered high concentrations of TPP (Tables 1 and 2).

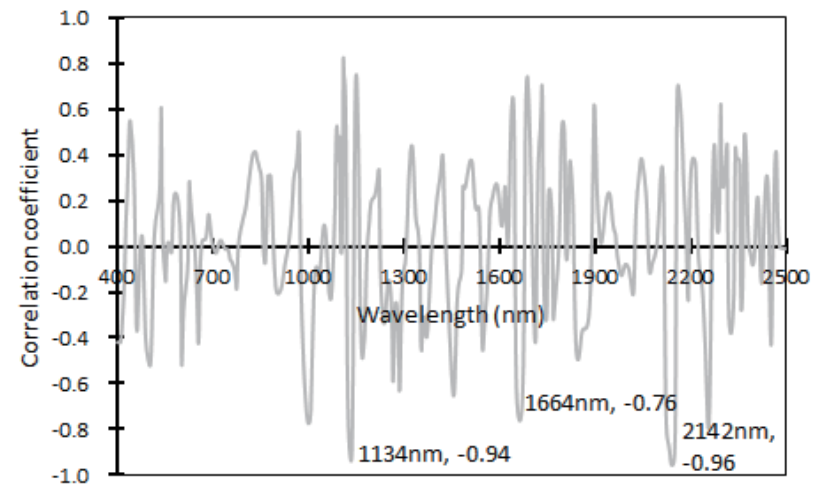

Fig. 7. Correlation coefficients between reference TPP content and SG2D values with SNV processing of raw spectra.

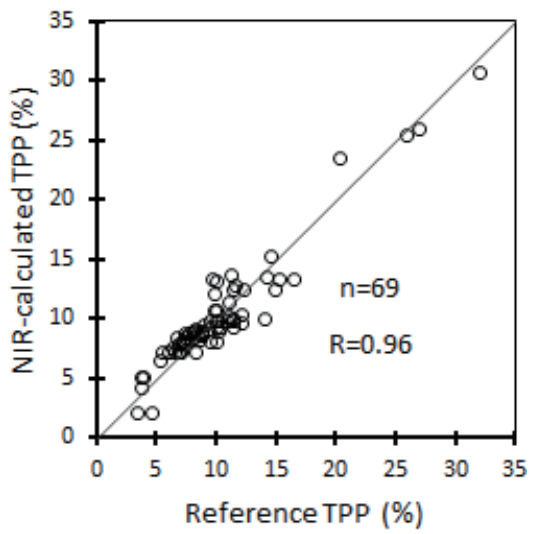

Fig. 8. Plots of reference TPP vs TPP calibrated at $2142 \mathrm{~nm}$ for SG2D with SNV. 


\subsection{Vis-NIR spectra}

The tea extract and tea extract containing dextrin had relatively sharp absorption peaks at $2140 \mathrm{~nm}$; both extracts, as well as the catechin mixture reagent, showed high reference TPP concentrations (Fig. 2). Thus, SG2D with SNV of the raw spectra may reduce the systematic noise in the spectra and enhance the contribution of the chemical components with lower 2D values. The relative order of their values (absorption) at 1660 and $2142 \mathrm{~nm}$ is Houji $<$ Kamairi $<$ Sunrouge $<$ tea extract containing dextrin $<$ tea extract, corresponding to the reference TPP concentrations [Figs. 3(b) and 4(b)].

\subsection{Optimal wavelength for predicting TPP content}

We carried out this study with the new ideas mentioned. We have developed numerous determination methods using NIR spectroscopy. As described in Sect. 1, calibration with various types of samples and a wide range of reference values leads to the derivation of more universal and accurate results, ${ }^{(17,25)}$ and the limits of the method may also be found. In addition, we attempted to measure Vis-NIR spectra in not the reflectance ${ }^{(7,8,10,11,14)}$ or diffuse reflectance mode ${ }^{(9,12,13,15,16)}$ but the transmittance-reflectance mode. Also, the older PLS analyses were simple as they did not involve SR or exhaustive analyses in combination with wavelength regions and preprocessing methods, and it was difficult to specify a few wavelengths. ${ }^{(7-16)}$

In this study, the NIR wavelength range of 2000-2498 nm, which has high sensitivity to TPP content, was selected for the best PLS model for predicting TPP content in various teas and tea extract (Table 3). Then, the raw spectra were preprocessed using SG2D with SNV. As described in the results, the regression coefficients and SR values of the best PLS model [Figs. 6(a) and 6(b)] suggest that the intensity around $2144 \mathrm{~nm}$ is almost proportional to TPP content, corresponding to lower 2D values. Therefore, the correlation coefficients between the reference TPP content and SG2D with SNV were investigated. SG2D with SNV gives a negative correlation coefficient when the systematic noise of the spectra is reduced and the contribution of the chemical component is enhanced. Then, the lowest negative $R$ value $(-0.96, n=69)$ was observed at $2142 \mathrm{~nm}$ in the wavelength range of 400 to $2498 \mathrm{~nm}$ (Fig. 7).

As mentioned above, an optimal wavelength of $2142 \mathrm{~nm}$ was identified for predicting TPP content in various teas and tea extract based on the regression coefficients, the SR values of the best PLS model, the peaks of the raw spectra and their SG2D with SNV, and the R values between the reference TPP content and SG2D with SNV of the raw spectra (Table 4).

Bian et al. reported that the intensities at 1131, 1654, 1666, 1738, and $1752 \mathrm{~nm}$ are related to TPP absorption. ${ }^{(10)}$ Moreover, they reported that the wavelength of $1648 \mathrm{~nm}$ can be linked

Table 4

Important wavelengths for prediction of TPP content in teas and tea extract.

\begin{tabular}{|c|c|c|c|c|}
\hline $\begin{array}{c}\text { Peak top of raw } \\
\text { spectra }\end{array}$ & $\begin{array}{l}\text { Peak top of } 2 \mathrm{nd} \\
\text { derivative values } \\
\text { (SG2D with SNV) }\end{array}$ & $\begin{array}{l}\text { Regression coefficient } \\
\text { of best PLS model }\end{array}$ & $\begin{array}{c}\text { SR value of best PLS } \\
\text { model }\end{array}$ & $\begin{array}{l}\text { Correlation coefficient } \\
\text { between reference } \\
\text { TPP and SG2D } \\
\text { with SNV }\end{array}$ \\
\hline $2140 \mathrm{~nm}$ & $2142 \mathrm{~nm}$ & $2144 \mathrm{~nm}$ & $2144 \mathrm{~nm}$ & $2142 \mathrm{~nm}$ \\
\hline
\end{tabular}


to the absorption feature of phenolic acid for tea powder and leaves. ${ }^{(11)}$ In Fig. 7, our results also showed a high correlation $(-0.94)$ at $1134 \mathrm{~nm}$ and a lower correlation $(-0.76)$ at $1664 \mathrm{~nm}$ between reference TPP and SG2D with SNV. Thus, we can use a wavelength of around $1134 \mathrm{~nm}$ for TPP determination. In the NIR region, the sensitivity to TPP content increased toward high wavelengths, as shown in Fig. 2, and we can observe only small absorbance at $1134 \mathrm{~nm}$ not only in the raw spectra (Fig. 2) but also in SG2D with SNV [Figs. 3(b) and 4(b)]. However, the wavelength of $2142 \mathrm{~nm}$ selected in the present study shows the highest $\mathrm{R}$ and has not been reported in previous studies. In addition, we show in Fig. 2(b) that the strongest absorption of the catechin mixture reagent (powder) is around $2142 \mathrm{~nm}$ in the NIR region.

The powder of a standard reagent can be measured to visualize the absorption band; sucrose, glucose, and fructose have been measured for the non-destructive determination of soluble solids in some fruits (melons, tomatoes, compact watermelons, strawberries) using NIR spectroscopy. ${ }^{(17,26)}$ The absorption band of the three sugars can be measured from 900 to $925 \mathrm{~nm}$ in the second derivative spectra, ${ }^{(26)}$ and the combination of wavelengths around $902 \mathrm{~nm}$ and other several wavelengths can be used for the practical and non-destructive determination of soluble solids in fruits. ${ }^{(17)}$ The main components of the soluble solids are these sugars. The absorption between 880 and $915 \mathrm{~nm}$ is generally assigned to the third overtone of the $\mathrm{CH}$ stretching vibration mode. ${ }^{(27)}$

Combination bands appear in the NIR region of 1900 to $2500 \mathrm{~nm} .{ }^{(27)}$ According to a quantum chemical calculation method, methanol in $\mathrm{CCl}_{4}$ has a combination of the $\mathrm{OH}$ stretching mode with the $\mathrm{CO}$ stretching mode at around $2151 \mathrm{~nm}\left(4650 \mathrm{~cm}^{-1}\right)$, although the peak is weak. ${ }^{(28)}$ This may lead to the assignment of $\mathrm{OH}$ stretching and $\mathrm{CO}$ stretching at around $2142 \mathrm{~nm}$ because both vibration modes can originate from alcohols or phenols and have strong absorption. The wavelength range of $3420-3250 \mathrm{~cm}^{-1}$ is assigned to $\mathrm{OH}$ stretching, having broad bands due to hydrogen bonds in alcohols and phenols (solids and liquids), and the wavelength range of $1340-1160 \mathrm{~cm}^{-1}$ is assigned to CO stretching, having broad bands in phenols as shown in Fig. 2. On the other hand, according to the absorption of phenol in $\mathrm{CCl}_{4}$, the wavelengths around $1134 \mathrm{~nm}$ correspond to the second overtone of the aromatic $\mathrm{CH}$ stretching vibration mode although the intensity is weak as shown in Figs. 2, 3(b), and 4(b). ${ }^{(27)}$

\section{Conclusions}

As a reference method for determining TPP content, the colorimetric ferrous tartrate method was applied and the absorbance of each sample blank was subtracted. Vis-NIR spectra of various teas and tea extract were measured in a new transmittance-reflectance mode. Also, the spectrum of a catechin mixture reagent was measured. PLS regression analyses were performed in combination with different wavelength ranges and spectral pretreatments. The best PLS model (2000-2498 nm) for predicting TPP content in dry teas and tea extract gave a wide range of TPP concentrations with high accuracy $(R=0.98)$. Moreover, absorption at $2142 \mathrm{~nm}$ was identified as the optimal independent variable based on regression coefficients, SRs of the best model, Vis-NIR spectra, and a correlation spectrum. This wavelength can be recommended for the rapid and accurate quantification of TPP content in teas and tea extract with high sensitivity in the NIR region. 


\section{Acknowledgments}

This work was supported by United Nations University-Kirin Fellowship.

\section{References}

1 K. Ozaki and S. Kawada: Measurement method series 32, Near-Infrared Spectroscopy (Japan spectroscopy society, Japan scientific societies press, Tokyo, 1996) 1st ed., p. 157.

2 M. Maeda-Yamamoto, K. Ema, M. Monobe, Y. Tokuda, and H. Tachibana: J. Agric. Food Chem. 60 (2012) 2165. https://doi.org/10.1021/jf204497b

3 M. Maeda-Yamamoto: Curr. Pharm. Des. 19 (2013) 6148. https://www.eurekaselect.com/115114/article

4 S. Masuda, M. Maeda-Yamamoto, S. Usui, and T. Fujisawa: Allergol. Int. 63 (2013) 211. https://doi.org/10.2332/ allergolint.13-OA-0620

5 M. S. Pinto: Food Res. Int. 53 (2013) 558. https://doi.org/10.1016/j.foodres.2013.01.038

6 K. Iwasa, I. Ota, and H. Torii: Chagyo Kenkyu Hokoku (Tea Res. J.) 33 (1970) 69 (in Japanese with English summary). https://doi.org/10.5979/cha.1970.33 69

7 H. Schulz, U. H. Engelhardt, A. Wegent, H. H. Drews, and S. Lapczynski: J. Agric. Food Chem. 47 (1999) 5064. https://doi.org/10.1021/jf9813743

8 Q. Chen, J. Zhao, X. Huang, H. Zhang, and M. Liu: Microchem. J. 83 (2006) 42. https://doi.org/10.1016/ j.microc.2006.01.023

9 V. R. N. Sinija and H. N. Mishra: Fresh Produce 3 (2009) 76.

10 M. Bian, A. K. Skidmore, M. Schlerf, T. Fei, Y. Liu, and T. Wang: Photogramm. Eng. Remote Sens. 76 (2010) 1385. https://doi.org/10.14358/PERS.76.12.1385

11 M. Bian, A. K. Skidmore, M. Schlerf, T. Wang, Y. Liu, R. Zeng, and T. Fei: ISPRS J. Photogramm. Remote Sens. 78 (2013) 148. https://doi.org/10.1016/j.isprsjprs.2013.02.002

12 X. Wang, J. Huang, W. Fan, and H. Lu: Anal. Methods 7 (2015) 787. https://doi.org/10.1039/c4ay02106a

13 J. Luypaert, M. H. Zhang, and D. L. Massart: Anal. Chim. Acta 478 (2003) 303. https://doi.org/10.1016/S00032670(02)01509-X

14 M. S. Lee, Y. S. Hwang, J. Lee, and M. G. Choung: Food Chem. 158 (2014) 351. https://doi.org/10.1016/ j.foodchem.2014.02.127

15 G. Ren, S. Wang, J. Ning, R. Xu, Y. Wang, Z. Xing, X. Wan, and Z. Zhang: Food Res. Int. 53 (2013) 822. https://doi.org/10.1016/j.foodres.2012.10.032

16 N. Panigrahi, C. S. Bhol, and B. S. Das: J. Food Eng. 190 (2016) 101. https://doi.org/10.1016/j.jfoodeng.2016.06.020

17 H. Ito: Jpn. Agric. Res. Q. 48 (2014) 111. https://doi.org/10.6090/jarq.48.111

18 W. Pan, J. Zhao, Q. Chen, and D. Zhang: Food Anal. Methods 8 (2015) 749. https://doi.org/10.1007/s12161-0149954-Z

19 plsropt package: https://github.com/uwadaira/plsropt

20 K. Iwasa and H. Torii: Chagyo Kenkyu Hokoku (Tea Res. J.) 19 (1962) 104 (in Japanese with English summary). https://doi.org/10.5979/cha.1962.104

21 R. J. Barnes, M. S. Dhanoa, and S. J. Lister: Appl. Spectrosc. 43 (1989) 772. https://doi. org/10.1366/0003702894202201

22 A. Savitzky and M. J. E. Golay: Anal. Chem. 36 (1964) 1627. https://doi.org/10.1021/ac60214a047

23 F. A. O. Olgun, D. Ozyurt, K. I. Berker, B. Demirata, and R. Apak: J. Sci. Food Agric. 94 (2014) 2401. https:// doi.org/10.1002/jsfa.6569

24 A. S. Roslan, Y. Ando, A. Azlan, and A. Ismail: Pertanika J. Trop. Agric. Sci. 42 (2019) 545.

25 H. Ito: Bull. Natl. Inst. Vegetable Tea Sci. 6 (2007) 83 (in Japanese with English summary, figures and tables).

26 H. Ito: Acta Hortic. 567 (2002) 751. https://doi.org/10.17660/ActaHortic.2002.567.165

27 J. M. Chalmers and P. R. Griffiths (Eds.): Handbook of Vibrational Spectroscopy 3 (John Wiley \& Sons Ltd, Chichester, 2002) pp. 1783, 1817.

28 K. B. Bec', Y. Futami, M. J. Wo' jcik, and Y. Ozaki: Phys. Chem. Chem. Phys. 18 (2016) 13666. https://doi. org/10.1039/c6cp00924g 